\title{
Wolff, Reinmar (Hrsg.): New York Convention, 2. Aufl., München, 2019. XXIII und 721 Seiten. ISBN 978-3-406-71445-0
}

\author{
Christian Armbrüster
}

Online publiziert: 26. Mai 2020

(C) Der/die Autor(en) 2020

Das Schiedsverfahrensrecht ist auch für die Privatversicherung von einiger Bedeutung. Dies gilt insbesondere für die Industrieversicherung und für die Rückversicherung. Schiedsverfahren bieten ein probates Mittel, anstelle der Streitentscheidung durch staatliche Gerichte ein nichtstaatliches, weitgehend privatautonom gestaltbares Verfahren zur Streitbeilegung zu nutzen. Die Anforderungen an eine wirksame Schiedsvereinbarung sind niedrig; ${ }^{1}$ verständigen sich die Parteien auf eine solche Vereinbarung, so sind sie für die Beilegung von Konflikten aus dem jeweiligen Rechtsverhältnis, auf das sich die Schiedsabrede bezieht, im Grundsatz nicht mehr auf Mitwirkung staatlicher Gerichte angewiesen. Allerdings gibt es zwei wichtige Ausnahmen: Soll eine Beweisaufnahme stattfinden, etwa indem im schiedsgerichtlichen Verfahren Zeugen vernommen werden, so bedarf es zur Erzwingung dieser Beweisführung unter Umständen einer Mitwirkung staatlicher Gerichte gem. §1050 ZPO. Zudem ist staatliche Hilfe immer dann erforderlich, wenn ein Schiedsspruch vollstreckt werden soll. Diesem Thema der Anerkennung und Vollstreckung von Schiedssprüchen widmet sich die New York Convention von 1958, die den Gegenstand des anzuzeigenden, nunmehr in 2. Auflage erschienenen Buches bildet.

Die „United Nations Convention on the Recognition and Enforcement of Foreign Arbitral Awards“, wie die New York Convention mit vollständigem Titel heißt, ist eine der erfolgreichsten UN-Konventionen auf dem Gebiet des Wirtschaftsrechts. Im März 2020 hatten die Konvention 163 Staaten unterzeichnet, darunter 158 der 193 UN-Mitgliedstaaten. Die zentralen Regelungen betreffen die Voraussetzungen, un-

\footnotetext{
1 Näher Armbrüster, VP 12-2019/01-2020, S. $12 \mathrm{ff}$.

C. Armbrüster $(\square)$

Fachbereich Rechtswissenschaft, Freie Universität Berlin, Van’t-Hoff-Str. 8, 14195 Berlin,

Deutschland

E-Mail: c.armbruester@fu-berlin.de
} 
ter denen ein Unterzeichnerstaat einen ausländischen Schiedsspruch anerkennen und durch seine eigenen Organe vollstrecken muss. Angesichts dessen, dass die Konvention in den meisten wirtschaftlich bedeutsamen Staaten der Welt gilt, liegt darin ein erheblicher Vorteil gegenüber den Urteilen staatlicher Gerichte, deren Vollstreckbarkeit in Staaten außerhalb des EWR von entsprechenden bi- oder multilateralen völkerrechtlichen Abkommen abhängt. Man kann nun speziell für den Versicherungssektor feststellen, dass die Vollstreckbarkeit hier - jedenfalls dann, wenn es um die Vollstreckung gegen einen Versicherer oder Rückversicherer geht - meist weniger problematisch erscheint als bei vielen anderen Parteien. Indessen gewinnen auch im Versicherungsbereich Schiedssprüche gerade dadurch ihre Autorität, dass sie in vollstreckungsrechtlicher Hinsicht Urteilen gleichstehen und dass sie im Vergleich zu letzteren international leichter vollstreckbar sind.

Das von Reinmar Wolff (Universität Marburg) herausgegebene und von ihm sowie neun weiteren Autoren bearbeitete Werk erläutert in englischer Sprache, aber in der Art deutscher Kommentare zu juristischen Regelwerken, die Artikel der Konvention. Der praktischen Bedeutung entsprechend liegen Schwerpunkte auf Art. II (Anerkennung von Schiedsabreden) und insbesondere Art. V (Gründe für die Ablehnung von Anerkennung und Vollstreckung von Schiedssprüchen). Dabei tritt an verschiedener Stelle zutage, vor welche Herausforderungen der Wortlaut des Textes von 1958 den heutigen Rechtsanwender bisweilen stellt. So verlangt etwa Art. II(2), dass die Schiedsabrede für ihre Wirksamkeit in Schriftform (,,in writing“) getroffen sein muss, was aus heutiger Sicht übermäßig streng erscheint. Demgemäß plädiert Wolff (Art. II Rn. 3, 78 ff., 118 ff.) mit sorgfältiger Begründung für eine an den Formzwecken orientierte, großzügigere Auslegung, deren Grundgedanke auch in $§ 1031$ Abs. 1 ZPO zum Ausdruck kommt.

Bei der Kommentierung zu Art. V füllt allein das eng gedruckte Schrifttumsverzeichnis nahezu vierzehn Seiten (S. 231-244). Es verwundert nicht, dass über die Voraussetzungen einer Anerkennung und Vollstreckung in der Praxis bisweilen hart gerungen wird, geht es dabei letztlich doch darum, wie weit die Bindungswirkung der Konvention reicht. Ein wichtiges Grundprinzip lautet, dass ein Schiedsspruch nicht auf seine inhaltliche Richtigkeit (,on the merits“) überprüft werden darf. Allerdings gibt es zwei praktisch bedeutsame Fälle, die jedenfalls de facto doch zu einer gewissen inhaltlichen Kontrolle führen, nämlich der Verstoß gegen den ordre public und in gewisser Hinsicht auch derjenige gegen das Gebot des fairen Verfahrens, wenn etwa gerügt wird, dass die Gewährung rechtlichen Gehörs verletzt worden sei, indem wichtiger Parteivortrag in den Gründen des Schiedsspruchs keinen Niederschlag gefunden habe.

Borris und Hennecke (Art. V Rn. 1 ff.) geben einen guten Überblick zu den Grundlagen der Ablehnungsgründe; sodann widmen sich Scherer (Art. V Rn. $128 \mathrm{ff}$.) dem fairen Verfahren und Wolff (Art. V Rn. $480 \mathrm{ff}$.) dem ordre public. Dabei wird jeweils deutlich, dass eine zu großzügige Handhabung der Ablehnungsgründe dem Geist der Konvention zuwiderlaufen und damit auch das Vertrauen in die Zuverlässigkeit des jeweiligen Staates, dessen Gerichte über eine Ablehnung entscheiden, untergraben würde. Zwar sind in weltweiter Sicht die Standards des ordre public weniger weitreichend harmonisiert als innerhalb der EU, in dem der ordre public-Vorbehalt des Art. 45 Abs. 1a EuGVVO n.F. bei der Vollstreckung von Entscheidungen der 
Gerichte anderer Mitgliedstaaten mittlerweile nur noch in besonderen Ausnahmefällen zum Zuge kommen kann. ${ }^{2}$ Gleichwohl gilt es auch bei der Anwendung der UN-Konvention sicherzustellen, dass das Verbot einer révision au fond nicht durch eine zu großzügige Annahme von ordre public-Verstößen ausgehebelt wird.

Auch die weiteren Gründe, die einen Unterzeichnerstaat berechtigen die Anerkennung und Vollstreckung abzulehnen, werden sorgfältig dargelegt. Insgesamt besticht das Werk auch in der Neuauflage durch übersichtlich und stringent aufgebaute Kommentierungen, die auch den Normzweck jeweils deutlich herausarbeiten und die praktische Arbeit mit der Konvention durch gut ausgewählte Nachweise zur weltweiten Rechtsprechung sowie durch übersichtliche Anhänge erleichtern.

Funding Open Access funding provided by Projekt DEAL.

Open Access Dieser Artikel wird unter der Creative Commons Namensnennung 4.0 International Lizenz veröffentlicht, welche die Nutzung, Vervielfältigung, Bearbeitung, Verbreitung und Wiedergabe in jeglichem Medium und Format erlaubt, sofern Sie den/die ursprünglichen Autor(en) und die Quelle ordnungsgemäß nennen, einen Link zur Creative Commons Lizenz beifügen und angeben, ob Änderungen vorgenommen wurden.

Die in diesem Artikel enthaltenen Bilder und sonstiges Drittmaterial unterliegen ebenfalls der genannten Creative Commons Lizenz, sofern sich aus der Abbildungslegende nichts anderes ergibt. Sofern das betreffende Material nicht unter der genannten Creative Commons Lizenz steht und die betreffende Handlung nicht nach gesetzlichen Vorschriften erlaubt ist, ist für die oben aufgeführten Weiterverwendungen des Materials die Einwilligung des jeweiligen Rechteinhabers einzuholen.

Weitere Details zur Lizenz entnehmen Sie bitte der Lizenzinformation auf http://creativecommons.org/ licenses/by/4.0/deed.de.

\footnotetext{
2 Siehe dazu etwa EuGH EuZW 2016, 713 m. Anm. Mäsch.
} 